\title{
Enseñanza y Aprendizaje Clínico del Derecho (EACD) - Investigación: integración para la educación jurídica*
}

\author{
Clinical Law Teaching and Learning (CLTL) - Research: \\ integration for legal education
}

\author{
Jorge Eduardo Vásquez Santamaría ${ }^{a}$ Ángela María Restrepo Jaramillo ${ }^{b}$ \\ ${ }^{a}$ Docente investigador del grupo de investigación Jurídicas y Sociales de la Facultad de Derecho y Ciencias \\ Políticas de la Universidad Católica Luis Amigó, Colombia. jorge.vasquezsa@amigo.edu.co \\ ${ }^{b}$ Magíster en Educación y Desarrollo Humano de la Universidad de Manizales, Colombia. \\ Docente de la Maestría en Ciencias: Innovación en Educación del Instituto Tecnológico Metropolitano. \\ angelarestrepo@itm.edu.co
}

\section{RESUMEN}

Se postula la EACD como un enfoque pedagógico que sin desconocer los legados de las corrientes y los modelos que le anteceden, procura trascender las elaboraciones que sustentaron la creación de Clínicas Jurídicas (CJ) como expresión de enseñanza y aprendizaje práctico del Derecho. Para ello se propone reposicionar la investigación en el campo jurídico como función generadora de conocimiento y fundamento de las razones, formas y potencialidades que motivan su integración con la EACD. Ésta última deja de asumirse como un modelo práctico de enseñanza y se retoma como un enfoque pedagógico abiertamente compatible con la función sustantiva de la investigación. Para sustentar la EACD como un enfoque pedagógico se propuso un diseño metodológico cimentado en el paradigma cualitativo, y desde el enfoque histórico hermenéutico se acude a fuentes documentales sobre las cuales se despliega el análisis de los datos cualitativos orientados a través de un proceso de categorización.

Palabras claves: Clínica Jurídica, Modelo Pedagógico, Enfoque Pedagógico, Método Clínico.

\begin{abstract}
The EACD is postulated as a pedagogical approach that, without ignoring the legacies of the currents and the models that precede it, seeks to transcend the elaborations that supported the creation of Legal Clinics (LC) as an expression of teaching and practical learning of Law. For this, it is proposed to reposition research in the legal field as a function that generates knowledge and the basis of the reasons, forms and potentials that motivate its integration with the EACD. The latter ceases to be assumed as a practical teaching model and is resumed as a pedagogical approach openly compatible with the substantive function of research. To support the EACD as a pedagogical approach, a methodological design based on the qualitative paradigm was proposed, and from the historical hermeneutical approach, documentary sources are used on which the analysis of the qualitative data oriented through a categorization process is deployed.
\end{abstract}

Key words: Legal Clinic, Pedagogical Model, Pedagogical Approach, Clinical Method.

\footnotetext{
* Artículo resultado del proyecto de investigación "Repensando la formación de los profesionales del Derecho. Hacia la construcción de una propuesta pedagógica pertinente para los programas de Derecho de Colombia", avalado en la IX convocatoria interna del Centro de Investigaciones de la Universidad Católica Luis Amigó; adscrito a la línea de investigación "Derecho y Sociedad" del grupo de investigación Jurídicas y Sociales de la Facultad de Derecho y Ciencias Políticas de la Universidad Católica Luis Amigó, Medellín, Colombia.
} 
Estudios Pedagógicos XLVII Nº 1: 431-451, 2021

ENSEÑANZA Y APRENDIZAJE CLÍNICO DEL DERECHO (EACD) - INVESTIGACIÓN: INTEGRACIÓN PARA LA EDUCACIÓN JURÍDICA

\section{INTRODUCCIÓN}

Desde el 2008 en varias universidades de Colombia se presentó un aumento en las iniciativas de creación de CJ en Facultades de Derecho. Experiencias destacadas en la Universidad del Rosario y en la Universidad de Medellín amplificaron los debates y las investigaciones que en adelante abrieron espacios de análisis sobre la incorporación de una nueva modalidad para emprender los procesos de enseñanza y aprendizaje del Derecho. Desde entonces la Facultad de Derecho y Ciencias Políticas de la Universidad Católica Luis Amigó ha hecho parte del debate. Su punto de partida es una investigación monográfica ${ }^{1}$ realizada en la Especialización en Docencia Investigativa Universitaria que buscaba proponer estrategias de mejoramiento en los procesos de formación promoviendo la cualificación del programa de Derecho. Luego, en la IX convocatoria interna de proyectos del Centro de Investigaciones de la Universidad Católica Luis Amigó fue aprobada la investigación "Repensando la formación de los profesionales del Derecho. Hacia la construcción de una propuesta pedagógica pertinente para los programas de Derecho de Colombia", que aborda el problema: ¿Qué elementos deben constituir una propuesta pedagógica pertinente para la formación de los profesionales del Derecho?

Entre los resultados de la indagación, la naturaleza y función de los Consultorios Jurídicos se convirtieron en la plataforma para fundamentar la creación y funcionamiento de las CJ como expresión de lo que se concibió como un modelo de formación práctica del Derecho, y si bien en la actualidad las CJ por regla general mantienen la naturaleza de ser espacios extracurriculares, muchas de ellas ahora son materias en los planes de estudio. No obstante, la Facultad de Derecho y Ciencias Políticas de la Universidad Católica Luis Amigó ve en los profundos cambios que definen la realidad colombiana un motivo para retomar los fundamentos que soportan la presencia y ejercicio de las CJ, enfatizando en la comprensión de su naturaleza pedagógica y en el reconocimiento de su potencial integrador de las funciones sustantivas de la educación superior, especialmente con la investigación. El debate se reposiciona en la EACD como base pedagógica sobre la cual reposan las CJ como la expresión más visible del que se considera un enfoque pedagógico con suficiencia y autonomía.

A la EACD se suma la reflexión que analiza el rol de la academia en las realidades sociales complejas que actualmente enfrentan el Estado y la Nación colombiana. El final de las negociaciones con las Fuerzas Armadas Revolucionarias de Colombia - FARC, la firma de un acuerdo para la finalización definitiva de la confrontación y la apertura del posacuerdo después de 50 años de conflicto armado interno son su insumo más inmediato.

Ese acontecimiento suscita un debate sobre los compromisos de la academia con la construcción de la paz en Colombia, toda vez que "Entre los sectores llamados a participar en este proceso la academia tiene un papel protagónico con la formulación de preguntas, escenarios de discusión, construcción de estrategias, entre otros, con el fin de aportar significativamente en este camino." (Pabón et al., 2016, p. 31). Adicionalmente, no puede dejarse de lado que en los años recientes el país atraviesa una crisis de corrupción y de funcionamiento del poder judicial, fenómenos frecuentemente asociados a la formación de los profesionales del Derecho.

"Evaluación de la producción científica de la Facultad de Derecho y Ciencias Humanas de la Fundación Universitaria Luis Amigó para la propuesta de planes de mejoramiento para la acreditación en calidad", ejecutada por Zonia Cristina Giraldo, Ana María Mesa Elneser y Jorge Eduardo Vásquez Santamaría. 
A su vez se suman los procesos de autoevaluación con fines de reacreditación en alta calidad de los programas de Derecho con el propósito último de asegurar su competitividad en el medio educativo, lo que ahonda las diferencias entre las instituciones que cuentan con el citado reconocimiento y las que no lo tienen. Especialmente la acreditación en alta calidad parece estar escindiendo los buenos y no tan buenos programas de formación en la educación superior, lo que explica Goyez (2010) genera "un mercado educativo en el cual sobreviven los más exitosos y eficientes" (p. 90).

Retomar los fundamentos de la EACD conlleva una reflexión sobre el enfoque pedagógico declarado, el ejecutado, y uno alternativo anhelado en las CJ; enfoque que es piedra angular de los registros calificados y las acreditaciones en alta calidad, metas que para este caso se conciben con el cumplimiento de requisitos sustanciales y no formales. Pero más allá de las dificultades y retos que propone la acreditación, en la investigación radica un enorme potencial para fortalecer y cualificar los procesos de enseñanza y aprendizaje. La investigación es el escenario que puede aprovechar la problematización de realidades vigentes no sólo para esclarecer los intereses y competencias del futuro abogado, sino para conducir y motivar a la aproximación y apropiación de la realidad para la cual se está formando. Ese atributo es el que puede y debe ser potenciado por la EACD, incluso para redefinir el fundamento pedagógico tradicional subyacente en los programas de Derecho, $\mathrm{y}$, además, tributar desde una perspectiva sustancial a la cualificación de los procesos de enseñanza y aprendizaje, y con ello, a la consecuente acreditación.

La EACD como una apuesta para fortalecer los procesos de enseñanza y aprendizaje ya no es novedosa en Colombia ni en Latinoamérica. Es el contexto instaurado con el posacuerdo lo que moviliza una fundamentación de la integración de procesos investigativos a la EACD, sin que aquellos remplacen o se sobrepongan al método clínico o a otros métodos activos. Se trata de retomar la naturaleza pedagógica de la EACD para ajustar su fundamentación y práctica pedagógica al contexto social cambiante y no reducirla a una parametrización del proceso de enseñanza y aprendizaje desplegado a la fecha.

La EACD respaldada en la investigación ostenta la fuerza para movilizar la reflexión y sentar las bases a partir de las cuales el servicio público educativo asuma su compromiso con la reconciliación y la construcción de la paz en un país que legitime el ejercicio de los profesionales del Derecho, pero ¿Por qué articular la investigación a la EACD? Para asumir "el liderazgo en una sociedad compleja, los universitarios no pueden pretender que lo que les brinda su facultad les es suficiente" (Böhmer, 2003, p. 4) y si no lo deben pretender los estudiantes de su Facultad menos lo deben hacer los docentes respecto a su experiencia frente a la universidad, pues, ¿qué brinda ésta en la actualidad a los actores pedagógicos del Derecho? La respuesta se concentra en una enseñanza y aprendizaje que sigue un ejercicio primordialmente memorístico, confiado en la norma, poco creativo, y alejado de los contextos sociales.

El proceso de formación en el saber jurídico continúa anclado en los fundamentos y prácticas del modelo pedagógico tradicional. Se trata de uno cuyo método es "el academicista, verbalista, que dicta sus clases bajo un régimen de disciplina, a unos estudiantes que son básicamente receptores" (Flórez, 1997, p. 170). Con tendencia a promover el acceso a contenidos acumulados de la ciencia mediante un método deductivo (Hernández, 2005) el tradicional perpetua el "protagonismo exclusivo del profesor, la relación vertical con los alumnos, el papel pasivo de estos, la memorización y el enciclopedismo como única exigencia (...)" (Minagawa, 2012, citado en Londoño, 2015, p. 10). Para el Derecho se 
trata de un proceso cifrado en la confianza en la codificación y en las normas jurídicas, aferrado al saber del docente que imparte saberes y omite la pregunta crítica y dinamizadora del conocimiento, pero sobretodo, que ve a la investigación como un escenario residual, distante y lejano, poco o nada necesario, en el que la capacidad de problematizar se suele promover, si es que se hace, en las aulas y alejada de la realidad social.

A partir de las exigencias y necesidades que se presentan a la educación superior se propone reposicionar la investigación no sólo como función generadora de conocimiento, sino como una determinante para fortalecer los procesos de enseñanza y aprendizaje. Por ello, la EACD deja de asumirse como un modelo práctico de enseñanza y se retoma como un enfoque pedagógico compatible con la función sustantiva de la investigación. Como enfoque pedagógico contribuye a la cualificación del proceso de formación, permite abordar y desarrollar nuevos objetos de investigación, sustentos epistemológicos y estructurar rutas metodológicas coherentes con las necesidades de los procesos de enseñanza y aprendizaje en una sociedad definida por nuevas realidades.

Para realizar ese cometido, se propuso un diseño metodológico cimentado en el modelo cualitativo y se aplica el enfoque histórico hermenéutico a fuentes documentales sobre las cuales se despliega el análisis de los datos cualitativos orientados a través de un proceso de categorización. Inicialmente se explica cómo la herencia del positivismo jurídico, especialmente del formalismo jurídico, permeó el ejercicio del Derecho, y con ello, determinó un modelo para su enseñanza y aprendizaje en Colombia. Posteriormente se da lugar a la figura de la EACD como enfoque pedagógico a través del cual se representa no sólo la reestructuración de la enseñanza y el aprendizaje del Derecho, sino una nueva comprensión de este. Luego, se explican las manifestaciones de ese enfoque pedagógico acudiendo a referentes que dan lugar a figuras propias de la EACD como enfoque y no como modelo de enseñanza y aprendizaje práctico del Derecho, como es el caso de las CJ y el método clínico.

\section{LA HERENCIA DEL DERECHO POSITIVO COMO DETONANTE DE LAS CLÍNICAS JURÍDICAS}

En Colombia el posacuerdo define la terminación de las expresiones del conflicto entre las FARC y el Gobierno Nacional, lo que no se traduce en su superación y en la realización de la paz social de forma automática. Pérez (2016) explica que los acuerdos no traen la inmediata realización de la paz, por el contrario, el posacuerdo define la terminación de una secuencia de exteriorizaciones del conflicto para dar lugar a un momento de transformación en el cual inician los trabajos para construirla. Coincide con Cepeda (2016) al ubicar en el posacuerdo la "fase posterior en la evolución del conflicto" (p. 195) y a partir de él se vislumbran las modalidades de paz que pueden venir después de la suscripción de los acuerdos.

La paz negativa pone el énfasis en la mera ausencia de guerra, de violencia directa. Presupone un aparato militar que garantice el orden, disuada al enemigo y asegure la perpetuación del statu quo (Asociación Pro Derechos Humanos 2000 citado en Pérez, 2016, p. 13). Pero la ausencia de guerra no concentra la garantía de paz, por lo que se debe buscar "la satisfacción de todas las necesidades humanas, incluyendo la 
realización de la justicia, el desarrollo económico y social equilibrado, la desaparición de la violencia estructural, la pobreza, la desigualdad, el racismo, la xenofobia, y los prejuicios y estereotipos culturales negativos" (Resolución 11.1. Conferencia General de la Unesco, 1974, citado en Pérez, 2016, p. 13).

Articulado al Estado de Derecho que requiere esta transición para la construcción de una paz positiva, y a todos los retos que de ello se derivan para la enseñanza y el aprendizaje de la abogacía, resulta imprescindible una fundamentación pedagógica que otorgue sustento a dichos procesos, no sólo en las exigencias de la transición dentro del marco de la seguridad jurídica, sino además, en la comprensión de las potencialidades de transformación histórica que radican en ella, y que demanda de los abogados capacidad para la superación del conflicto y para transformar la realidad en dirección a la convivencia pacífica. La necesidad imprescindible de una fundamentación pedagógica capaz de promover ese perfil del abogado no ha encontrado condiciones en el modelo tradicional. Lo anterior ratifica la urgencia de retornar a la cualificación de los procesos de enseñanza y aprendizaje del Derecho más allá de las prácticas y concepciones pedagógicas, para asumirla como un desafío sobre la forma de comprensión del Derecho.

El modelo pedagógico tradicional replicado en Colombia, sobre el cual se ha edificado la comprensión del Derecho, tiene una fuerte y perpetuada herencia del positivismo jurídico. Narrar el basto campo en el cual este ha sido trabajado desbordaría los propósitos de este texto, pero con el cometido de evidenciar su incidencia en el modelo pedagógico que ha operado en la formación de los abogados colombianos, es apropiada la siguiente caracterización. Desde Nino (2003) el positivismo jurídico puede ser definido como la corriente en la cual no hay una conexión o asociación entre el Derecho y la moral, descartando tesis como "Las normas de todo sistema jurídico reflejan de hecho valores y aspiraciones morales de la comunidad en la cual rigen (...) Las normas de un sistema jurídico deben ajustarse a ciertos principios morales y de justicia que son universalmente válidos" (p. 17) de tal manera que la validez de una norma no dependía de su correspondencia con principios de justicia.

Bobbio (2015) estima tres aspectos para distinguir el positivismo jurídico: como teoría del Derecho, como una ideología de la justicia, y "como un modo de acercarse al estudio del Derecho" (pp. 39-40). Desde el último aspecto el Derecho Positivo diferencia el Derecho como hecho del Derecho como valor, eliminando con ello los juicios de valor, y asegurando la objetividad con la abstención de toma de posición frente a la realidad aprehendida. Se desprende una postura avalorativa sin elementos finalistas. Esa neutralidad es la cuarta tesis propuesta por Prieto (2005) para caracterizar el Derecho Positivo. A esta postura avalorativa suma la premisa que el Derecho tiene su origen en la práctica social y no en ninguna otra parte, lo que hace del Derecho un hecho que está respaldado por el uso de la fuerza y al tiempo, regula y condiciona su uso, para finalmente incluir la inexistencia de la obligación moral de obediencia al Derecho.

La herencia de un Derecho elaborado y puesto, respaldado en la coerción, expresado a través de las normas jurídicas, y lejano a la valoración y la realización de cometidos finalistas, incide en la formación de abogados carentes de un pensamiento complejo y profundo en el que la realidad sea sustento necesario de un ejercicio permeado por el deber ser que exige la construcción de toma de posición. Desde el positivismo jurídico pervive la "insuperable cultura formalista del Derecho, a saber, la forma lógica y el silogismo práctico 
jurídico; segunda, la separación entre derecho, moral y política; y tercera, el elevado y aséptico análisis judicial” (López citado en Sarmiento, 2014, pp. 70-71). Esa tradición pervive en el modelo pedagógico tradicional contra el cual reacciona la EACD.

A pesar de ser sistemas jurídicos diferentes, el origen de la EACD en los Estados Unidos a inicios del siglo XX comparte en las características de la tradición jurídica la misma razón que motivó su aparición en Latinoamérica a finales del mismo siglo. Abramovich (1999) ubica el surgimiento de las CJ en los Estados Unidos a raíz de las críticas de Jeromy Frank en 1933 en su trabajo ¿ Why not a Clinical Lawyer School? en el cual recomienda acudir a la experiencia de la formación de la medicina cifrada en una práctica a partir de casos reales, debido a la concepción extremadamente positivista y logicista de la enseñanza y el aprendizaje del Derecho.

Desde Fergusson (2009) se aprecia que en Colombia aún impera un modelo de enseñanza y aprendizaje del Derecho históricamente formalista, centrado en la transmisión de información desde una relación unidireccional dirigida preponderantemente por los docentes frente a los estudiantes a través de clases magistrales. En ese modelo se promueve la memorización de contenidos teóricos con la finalidad de ser posteriormente aplicados y transmitidos a través de su repetición, hace "énfasis en la descripción y explicación de los contenidos normativos del sistema jurídico y en la doctrina acumulada al respecto, los cuales deben ser memorizados por los estudiantes" (Gómez, 2008, p. 107).

La información transmitida es principalmente jurídico formal, esto es, está sustentada en la forma de comprensión del Derecho primordialmente a partir del pensamiento ius positivista, enfocando el proceso de enseñanza y aprendizaje al estudio de la normativa con frecuentes remisiones a antecedentes históricos que en muchas ocasiones no evidencian una conexión para problematizar y comprender la finalidad de acudir a ellos para orientar la comprensión y aplicación del Derecho en la realidad actual. Se trata de la prevalencia del "estilo expositivo o magistral por parte del docente que describe los instrumentos legales vigentes, así como la jurisprudencia y la doctrina.” (Gómez, 2008, p. 107).

La estricta relación unidireccional entre docente y estudiante que define el modelo tradicional de enseñanza y aprendizaje del Derecho no es gratuita. La perpetuación de esta modalidad de interrelación está fuertemente incidida por dos componentes del modelo pedagógico tradicional: los contenidos y los métodos de enseñanza. A partir de las responsabilidades que recaen en lo que se gesta en el aula para la enseñanza y aprendizaje tradicional del Derecho, Böhmer (2003) propone dos preguntas: "qué se enseña y de qué manera se lo enseña" (p. 21). Frente a los contenidos la respuesta privilegia la herencia Napoleónica del Derecho, con lo que coincide Abramovich (1999) que considera que a raíz de la tradición romanista del Derecho continental en la enseñanza y aprendizaje del Derecho se ha puesto la atención en el estudio de los textos legales, relegando la práctica profesional y judicial. Abramovich (1999) explica que el legado del derecho romano a través de la compilación justinianea, y con ello, la reverencia acrítica de los juristas quedó plasmado en el Código Napoleónico que irradió las codificaciones civiles latinoamericanas vigentes.

De la posición predilecta de las normas jurídicas, la privilegiada ubicación del poder legislativo y de la Ley como su resultado máximo. En esa tradición los códigos elaborados por el legislador adquirieron un rol privilegiado que definió una tradición donde: "El rol de los jueces es aplicar el Código sin alterarlo, los intérpretes no deben existir. El abogado es simplemente un expositor del derecho que asiste a su cliente" (Böhmer, 2003, p. 23). 
En la enseñanza y aprendizaje del Derecho el código ha sido por excelencia un entramado de normas del cual aún se predica su orden, claridad y en ocasiones, como lo hizo el formalismo jurídico, su naturaleza completa con capacidad de abarcar cualquier situación, en el cual se localiza la norma jurídica como principal herramienta para comprender y aplicar el Derecho. De ello que la enseñanza y el aprendizaje del Derecho se haya concentrado en el estudio de la norma jurídica, y con ella, su memorización, exposición y repetición sin mayores pretensiones de interpretación, modificación o revaluación. Esta tradición es igualmente avalada por Bonilla (citado por Londoño, 2015, p. 9) quien la explica a partir del estudio de la ley en sentido formal, la mitificación de la separación de poderes, la separación entre el Derecho y la política, y la equiparación de la validez formal con la justicia.

El tradicionalismo en la enseñanza y aprendizaje del Derecho no sólo se definen por lo que se enseña, como se deduce de Böhmer (2005), sino también por la forma en cómo se hace. Ferguson (2009) destaca dentro de la historia de la educación jurídica colombiana la ausencia de aplicación de metodologías activas de aprendizaje, el encasillamiento en la normativa jurídica, el reduccionismo del concepto de aula, y con él, de todos los componentes o elementos que definen un modelo pedagógico para la enseñanza y el aprendizaje del Derecho.

Si históricamente al juez le estuvo vedado la posibilidad de interpretar las disposiciones jurídicas y ejercer un rol discrecional de cara a los hechos, resultaba igualmente remoto emprender la práctica hermenéutica dentro del aula de clase. Pero la tradición que permea la enseñanza y el aprendizaje del Derecho en relación a las formas de enseñanza aún transita por privilegiar la memoria: "demostrar que se puede repetir lo que dijo el docente en clase que es a su vez la repetición de lo que dice el manual, que repite lo que dice el Código" (Böhmer, 2003, p. 24).

\section{LA EACD: DE MODELO A ENFOQUE PEDAGÓGICO}

Antes de hacer explícito qué se asume por enfoque en el contexto de la formación de los abogados, y más en lo concerniente a la enseñanza y el aprendizaje, es importante considerar el uso recurrente de este término como sinónimo de modelo (Vásquez, 2008, 2012 y 2013, Cañaveral y Vásquez, 2010) para además explicitar el valor de transitar del modelo hacia el enfoque en el marco de la formación en el Derecho sin detrimento de los conceptos de base y de sus implicaciones en el acto pedagógico.

El modelo usualmente alude a un patrón particular (De Zubiría, 1999) representativo de una realidad, el cual por su precisión posibilita predictibilidad alrededor de lo representado por él; en la literatura es común encontrarlo también como paradigma y en este sentido "raramente es objeto de renovación" (Khun, 2004, p. 51) toda vez que "tienen más éxito que sus competidores para resolver unos cuantos problemas que el grupo de profesionales ha llegado a reconocer como agudos." (Khun, 2004, p. 52).

El modelo lleva implícita una alusión a la exactitud, la rigidez y la precisión para lograr el conocimiento de la realidad, con lo que se entiende desde Lakatos que "no se trata de conocer la realidad, sino de conocer el grado de precisión de los modelos diseñados para interpretarla o representarla, lo que en la práctica bien pudiera entenderse a partir de la formulación de hipótesis" (Gallego, 1999) y su consecuente verificación, lo 
que denota una concepción positivista subyacente al fenómeno atendido. No obstante, en el campo de la educación, y particularmente en el ámbito de lo pedagógico, sus objetos, los sujetos que en ella se infieren y sus rutas de abordaje, no dan lugar a consideraciones lineales y cerradas.

Hablar de modelos al pensar la educación sería contradictorio con lo variable, impredecible, diverso, circunstancial y lo complejo que le es propio. Las realidades educativas desbordan el anteponerse a la totalidad de lo que serán siempre que son susceptibles de fluctuaciones dependiendo de las circunstancias del momento. Con esto no se afirma que el hecho educativo no pueda ser intencionado; la planeación para su gestión es no sólo necesaria, sino que además es uno de los factores de éxito asociados a su calidad. No obstante, en su acontecer hay circunstancias, pero además retos y oportunidades que permiten salirse de los límites, superar las fronteras, explorar rutas complementarias y abrirse a nuevas y probablemente más pertinentes apuestas.

En este sentido, atender a la denominación de enfoque es oportuno para dar un giro a la mirada del asunto observado, en este caso del proceso para y en la formación de los abogados, en referencia a unos elementos o categorías previas, pero otorgándole a la vez la posibilidad de expandirse, de dialogar con las consideraciones de otros enfoques y de nutrirse de ellos sin perder su identidad, aquello que lo hace particular en coherencia con la intencionalidad formativa.

Desde esta perspectiva más que pensar modelos, buscaríamos acoger los enfoques, como sistemas orientadores para la comprensión y la puesta en acción en torno a un propósito educativo, constituidos por diversos elementos que sólo pueden entenderse y definirse en la complementariedad suscitada en sus interacciones y relaciones. Así, más allá del modelo, en el enfoque pedagógico como sistema subyacen según Behar, Passerino, y Bernardi (2007) "principios teóricos que representan, explican y guían la construcción e implementación del currículo y se materializan en las prácticas pedagógicas y en las interacciones maestro docente-objeto de conocimiento".

A su vez, el enfoque pedagógico se deriva de los lugares comunes entre teorías, escuelas o corrientes -corrientes pedagógicas-, que comparten sustratos epistémicos e intencionalidades propias en torno a la educación. De esta manera, si cada corriente piensa en los fundamentos teóricos para concebir y orientar los procesos de formación (Vásquez, 2012) en un contexto histórico cultural específico, la cercanía o la afinidad entre sus fundamentos y los de otras corrientes develará su coincidencia en un enfoque en cuya lógica interna se articularán concepciones del ideal de sociedad que se pretende tener: de los sujetos (estudiantes, alumnos, aprendices, etc.) que construirán o transformarán esa sociedad y de aquellos (docentes, profesores, instructores, tutores) que los formarán (educarán, instruirán, enseñarán, etc.). También del tipo de saberes (saber académico acumulado, problemas, ideologías, procesos cognitivos, etc.) de mediaciones (centradas en la enseñanza, en el aprendizaje, en la enseñanza y el aprendizaje, etc.) para la formación, y de las estrategias (clase magistral, centros de interés, investigación, experimentación, resolución de problemas, etc.) que darán cuenta de los aprendizajes obtenidos.

Visto en este contexto, más que afirmar la existencia de modelos pedagógicos, debería propenderse por validar la de enfoques pedagógicos, que perviven y que en ocasiones toman elementos unos de otros, bien sea desde las decisiones deliberadas de quienes piensan y ponen en acción el acto pedagógico, o desde sus actuaciones espontáneas -que darán cuenta de componentes fundamentales del currículo oculto-. 


\subsection{LAS CLÍNICAS JURÍDICAS: LA CARA MÁS VISIBLE DEL ENFOQUE PEDAGÓGICO}

Con base en la EACD como enfoque pedagógico, las CJ en Latinoamérica, y especialmente en Colombia, son la cara más visible del movimiento por medio del cual no sólo se cuestiona el modelo pedagógico tradicional de enseñanza y aprendizaje, sino, la forma de comprender el Derecho. Revalúan la herencia del formalismo jurídico, su incidencia en el ejercicio de los poderes públicos, y con ello, la fundamentación pedagógica e investigativa de las Facultades de Derecho. En Colombia la aparición de las CJ se identifica con "el cambio constitucional en 1991, gracias a la proclamación del Estado Social de Derecho, el reconocimiento de una amplia carta de derechos humanos y el establecimiento de las acciones constitucionales para su protección." (Castro, 2015, p. 74) paradigma constitucional que daría lugar a la transformación del pensamiento sobre la totalidad del orden jurídico imperante hasta la fecha.

Las CJ ponen en evidencia un enfoque pedagógico en construcción que surge de la anomalía que caracteriza no sólo el escenario de enseñanza y aprendizaje tradicional sino de sus prácticas profesionales. Las CJ son la expresión de una nueva corriente que promueve un cambio paradigmático que se traduce en el abandono de los componentes más incompatibles del modelo pedagógico tradicional con la realidad actual, y tras ello, del formalismo jurídico en la academia.

El nuevo enfoque se justifica y legitima a partir de las necesidades, recorridos y experiencias del modelo tradicional en una integración con teorías y corrientes que tienen en común la comprensión del Derecho también a partir de la realidad en la cual se recrea; de la acción y la participación de la sociedad, del equilibrio teórico-práctico, y de la investigación como componente transversal de formación.

Las CJ representan el esfuerzo por transitar de ese modelo de tipo heteroestructurante a un enfoque que se aproxime en su mayor posibilidad a uno autoestructurante. El tránsito implica superar la comprensión de la enseñanza y el aprendizaje a partir de una visión que la reduce a la figura de modelo, como estructura fija, definida y de poca variación, y más aún, de aquella que defiende como función esencial de la escuela "la de transmitir la cultura humana a las nuevas generaciones" (De Zubiría, 2006, p. 13). Como explica el autor, se trata de modelos magistrocentristas que defienden el uso de métodos receptivos donde predomina la enseñanza, la instrucción y el autoritarismo. Superar el modelo heteroestructurante implica abandonar la idea del estudiante como un sujeto pasivo que está supeditado a la información que recibe y a la orientación ofrecida por el maestro, de la enseñanza privilegiada como eje de acción, de los contenidos jerarquizados ofrecidos desde directrices externas que dejan de lado la motivación del estudiante, aspectos todos que compaginan con las prácticas pedagógicas más recurrentes del Derecho en Colombia.

Las CJ defienden la puesta en marcha de un enfoque pedagógico autoestructurante, que siguiendo a Not (1983) debe estar relacionado con las prácticas educativas que conciben la posibilidad del aprendizaje desde la actividad interna del sujeto, con preponderancia del descubrimiento, la construcción y los saberes previos, y la consideración del estudiante como protagonista con capacidad de participación, acción y transformación de su proceso.

Para las CJ los diversos componentes del acto pedagógico se determinan y delimitan en coherencia con estas concepciones, así el currículo define sus intencionalidades, propósitos y criterios de formación en la perspectiva de atender las necesidades, los intereses y las motivaciones del aprendiz; la didáctica se estructura y dinamiza a partir de la consideración 
sobre las capacidades y posibilidades del aprendizaje del estudiante, y la evaluación atiende a las características individuales del sujeto en formación y al seguimiento cualitativo de su desarrollo.

Para explicar el enfoque pedagógico que puede sustentar a una CJ se acude a cuatro preguntas propuestas por Coll (Citado por De Zubiría, 2006, p. 14) a partir de las cuales se busca puntualizar las intenciones y finalidades educativas de la EACD, preguntas que para nuestro caso se concretan como elementos constitutivos de un enfoque pedagógico y son esenciales para dar soporte a los componentes de la CJ. A partir de la pregunta ¿Qué enseñar? se representan los contenidos para la enseñanza y el aprendizaje del Derecho. En las CJ los contenidos no se conciben como asignaturas o cursos parcelados, delimitados, cerrados e inconexos. Por el contrario, se trata de saberes abiertos, relacionales, expansibles y respetuosos de las trayectorias y acumulados en armonía con las necesidades vigentes, escenarios dispuestos al encuentro y a la interacción de acuerdo a la naturaleza de las realidades seleccionadas para el estudio. De ello que las CJ no estandarice el proceso de formación dependiendo del número o tipo de cursos previamente abordados por el estudiante, o reduzca la experiencia de formación al control a partir de los prerrequisitos que establece el plan de estudio, en el sentido de exigir previamente la aprobación de ciertas materias para poder acceder al conocimiento de otras que se involucran en los casos abordados dentro del ejercicio clínico.

Los contenidos proponen la comprensión de un Derecho que lejos de ser dividido y parcelado, es holístico, global, integrador, pero sobretodo, dinámico e interdisciplinar, en el cual la realidad o acontecimientos que circulan en los procesos de enseñanza y aprendizaje, en una interacción equilibrada entre teoría y práctica, determinan el conjunto de saberes que deben confluir para dar respuesta a los problemas que se derivan de esa situación, los cuales trascienden al Derecho, llevándolo a diálogos con otros campos de conocimiento y otras metodologías.

Los contenidos tienen la capacidad de reunirse, encontrarse y confluir como potencialidades necesarias a través de las cuales se hace posible el estudio e indagación de una problemática social. No siguen una jerarquización o preferencia a partir de la denominada importancia que tienen dentro del modelo pedagógico tradicional, donde la herencia civilista y publicista privilegia asignaturas con contenidos claramente definidos en una de esas dos tendencias, y hace de otros temas asuntos complementarios, comúnmente secularizados por el plan de estudios y vistos con algo de indiferencia por los estudiantes y docentes.

Desde la pregunta ¿Cuándo enseñar? las CJ piensan y delimitan los momentos específicos para el acto pedagógico sin aferrase a la estructura estandarizada y en ocasiones inamovible de los programas tradicionales, sino en atención a los requerimientos y demandas que plantean los problemas y los objetos de estudio que de ellos se derivan. Lo anterior conduce a que en la CJ no se pospone el abordaje y aprehensión de un objeto de estudio por el nivel en el que el programa ubica al estudiante, e incluso, en ocasiones, por su pertenencia a un programa diferente al de Derecho. De hacerlo, no sólo se está cerrando la puerta a la interdisciplinariedad que debe permear la enseñanza y el aprendizaje, sino que se perpetuaría la idea de incapacidad del estudiante e incluso del docente, en quienes prevalecería una idea de formación restrictiva y condicionada que responde a los órdenes tradicionales sobre los cuales se ha guiado la enseñanza y aprendizaje de lo jurídico.

Preguntarse por los momentos para la enseñanza y el aprendizaje del Derecho permite derivar del ejercicio de la CJ una característica cada vez más evidenciable desde 
las experiencias vigentes. El momento del aprendizaje progresivamente es asumido por el estudiante sin la necesaria supervisión del docente. El empoderamiento del rol de abogado frente a casos reales despierta en el estudiante un sentido de pertenencia, apropiación y responsabilidad que asegura la sensibilización con el objeto abordado y de paso, una ampliación progresiva de tiempo, de iniciativa individual, en la cual el estudiante es autogestor de sus labores frente al colectivo clínico y el cliente o usuario de las CJ. Cuando en una CJ se logra despertar tal sentido en un estudiante, los momentos e intensidades del aprendizaje alcanzan en ocasiones destinaciones muy superiores a las esperadas, y con ello, se gesta un cambio en la forma de comprender el Derecho a partir del hacer activo del aprendiz.

El componente evaluativo es uno de los más distintivos en el ejercicio de las CJ. Al ¿Qué, cuándo y cómo evaluar? las CJ ha elaborado una articulación de modalidades que no se contraponen entre si y enriquecen el aprendizaje a partir de la experiencia personal dentro de un grupo que promueve el trabajo colaborativo. La evaluación en las CJ suele ser preferentemente cualitativa, interna y externa.

Cualitativa en la medida que se suelen desarrollar estrategias en las cuales se descarta la idea de calificar al estudiante a través de un sistema cuantitativo cerrado que estandariza, esquematiza, segrega e incluso estigmatiza, en ausencia de orientaciones, críticas constructivas y recomendaciones para mejorar. La evaluación cualitativa acarrea la descripción, caracterización y valoración del proceso a partir de la experiencia del estudiante, exaltando las fortalezas de la persona en el ejercicio clínico, permitiendo su correlación con puntos de contraste, la retroalimentación, pero sobretodo, conocer las apreciaciones y reflexiones del estudiante como centro del proceso formativo.

De la evaluación interna se predica que esté integrada por la autoevaluación y la coevaluación. La primera está a cargo del estudiante como centro articulador del proceso de enseñanza y aprendizaje, en quien reposa el conjunto de experiencias vividas. Desde la segunda se expresan las valoraciones del docente acompañante o supervisor del proceso de enseñanza y aprendizaje, de otros docentes que de forma permanente u ocasional intervienen, y de los estudiantes que integran el grupo de trabajo, lo que fortalece la construcción de juicios de valor, el sentido ético que exige la responsabilidad de retroalimentar con franqueza y de forma constructiva el trabajo del otro, el trabajo en equipo, la disciplina, la identificación y comprensión de errores, y la capacidad de corregir y mejorar.

La evaluación externa se genera en la mayoría de los casos de manera casi obligada y sin ser requerida, pues es aquella que emiten usuarios y destinatarios de los servicios de las CJ, así como entidades, organismos y autoridades con los cuales se interactúa en casos concretos durante amplios periodos de tiempo. En este tipo de evaluación también se puede hacer partícipe la universidad quien debe velar por la gestión y sistematización del proceso de enseñanza y aprendizaje.

Sobre el interrogante ¿Para qué se enseña? se considera que ya se han ofrecido más arriba elementos que justifican la aparición y consolidación de las CJ en América, sin dejar pasar la posibilidad de sintetizar su finalidad en promover la formación de abogados a partir de un nuevo enfoque de enseñanza y aprendizaje en el que colaborativamente se reestructura la comprensión del Derecho como un campo de conocimiento que desde su formación pedagógica debe cumplir un rol efectivo frente a las demandas públicas de la sociedad.

Finalmente aprovecharemos una quinta pregunta propuesta por Coll para ahondar en la naturaleza y alcances de las CJ. Desde ¿Cómo se enseña? no sólo se estructura la interrelación entre el quién, el a quién y los contenidos, sino que se define la identidad 
de un enfoque pedagógico nuevo que tiene como cara más visible a las $\mathrm{CJ}$, y que desde la experiencia colombiana sistematizada por Londoño (2015, p. 63) en las Facultades de Derecho que la han acogido ha representado "aprendizaje activo, formación integral, aprendizaje constructivo, aprendizaje autorregulado, aprendizaje significativo, y aprendizaje - servicio".

\section{2. ¿CÓMO SE ENSEÑA? A PARTIR DE LA ENSEÑANZA CLÍNICA DEL DERECHO}

La EACD toma como referente la fundamentación de la enseñanza y el aprendizaje del profesional de la medicina. En ella, el estudiante es conducido de forma temprana en compañía de profesionales a escenarios reales donde tiene contacto directo con casos prácticos de distinta naturaleza, en los cuales resulta esencial la supervisión y orientación de un profesional docente que no suplanta al estudiante como eje del acto de aprendizaje y lo convierte en el centro principal de formación, a partir del cual también resultan nuevas pautas para la enseñanza.

Abramovich (1999) plantea que la EACD entraña un método particular de enseñanza, por medio del cual el estudiante asume un rol en una situación concreta y debe interactuar con los demás estudiantes para identificar los problemas de esa situación y proponer soluciones. Se trata de "una manera en la cual se pueda formar a estudiantes de derecho con parámetros que le permitan atender las demandas de su realidad social, política y económica" (Torres, 2013, p. 703), práctica que se puede ejercer a partir de casos simulados o reales, y finaliza con una evaluación grupal de quienes participaron en el ejercicio. Para Abramovich (1999) en la EACD se fusiona un aprendizaje a partir de la propia experiencia con uno colaborativo, para potenciar el poco tiempo que implican los años de formación en la Universidad, de tal manera que el estudiante aprenda a aprender de su experiencia personal.

Con la aproximación temprana del estudiante a la realidad inmediata que se constituirá en su escenario profesional se garantiza el contacto con los distintos elementos que justifican el proceso de enseñanza y aprendizaje, de los que depende su ejercicio profesional, y que permiten asegurar el cumplimiento del rol del respectivo campo científico en la sociedad; se trata de reequilibrar la formación teórico-práctica. El contacto directo con los pacientes en centros de atención le exige al estudiante implementar los conocimientos teóricos a través de prácticas concretas, dando lugar a una ininterrumpida experiencia personal que promueve un aprendizaje significativo guiado principalmente desde una formación teórico-práctica en la cual se potencia la interpretación y proposición de posibles soluciones a cada caso.

Para el estudiante de medicina, que en el proceso de enseñanza y aprendizaje asume el rol de médico, se hace esencial la implementación e indagación de los referentes a partir de los cuales debe realizar la aprehensión de cada caso y la solución al problema que encierra. Ese ejercicio demanda la comprensión preliminar de los hechos, acoger el testimonio y sentir del paciente, caracterizar la situación y formular un problema clínico que orientará el desempeño de sus acciones. Estas actividades no se hacen de manera aislada sino en compañía del supervisor o grupo de profesionales que acompañan el proceso de enseñanza y aprendizaje, y de los demás estudiantes de equipo, de los que se generan retroalimentaciones, apoyos, críticas constructivas y evaluaciones. Con ello el estudiante dimensiona una multiplicidad de variables que no son posibles de comprender si su estudio se reserva a los textos o referentes teóricos, libros de caso o fuentes científicas con resultados de investigación médica. 
La carga emotiva de cada situación, las tensiones y expectativas, la dificultad en los diagnósticos, la evaluación de los tratamientos y evolución de los pacientes, los debates entre los mismos profesionales frente a casos complejos, la variación entre un caso y otro, la intensidad en la evolución y desenvolvimiento de los tratamientos y las enfermedades, la presión del tiempo, de familiares y conocidos del paciente, hasta la forma en cómo se tiene que asumir una pérdida o error en el ejercicio, constituyen elementos que difícilmente llegan a ser puestos en conocimiento del estudiante dentro del acto pedagógico adscrito a un modelo tradicional. Como explica Duarte (2012) en la enseñanza clínica de la medicina

la creación de un clima de aprendizaje estimulante, la evaluación de los estudiantes en la práctica, principalmente a través de la observación directa de sus desempeños, la provisión de devoluciones significativas y el compromiso en la reflexión son los principales componentes de este modelo (p. 149).

Con base en la experiencia de la enseñanza y aprendizaje de la medicina, en los países de herencia jurídica continental, la EACD asume la experiencia práctica como alternativa pedagógica relevante, la cual es frecuentemente desechada por los métodos tradicionales de educación legal (Correa y Vásquez, 2009). Evitar la escisión de la práctica y la teoría es un cometido irrenunciable de la EACD, toda vez que con ello se evita el desconocimiento del trasfondo social que nutre al Derecho como ciencia, razón por la cual uno de sus más grandes desafíos radica en alcanzar el equilibrio teórico-práctico.

En ese esfuerzo, la EACD no persigue desechar el legado de la educación jurídica impartida de forma tradicional (Correa y Vásquez, 2009) pero si superar sus principales elementos reduccionistas y desarticulados de las necesidades contemporáneas. La EACD pretende superar la actividad de transmitir, memorizar y repetir el texto normativo como fuente prioritaria de información jurídica impartida al interior de un aula desprendida de las dinámicas sociales. Desmitifica la reverencia de la codificación, del fundamento teórico y del temor al disentimiento; del rol del docente como eje activo del acto pedagógico y del estudiante como ente pasivo receptor de conocimientos incontrovertibles, preponderantemente teóricos y parcelados; del autoritarismo y del espíritu absolutista del maestro, y con ello, de la relación de verticalidad frente al estudiante.

La EACD subsana la ausencia del aprendizaje práctico inmerso en realidades vigentes, y el aislamiento y falta de contacto con quienes padecen las problemáticas que requieren de la atención del abogado como profesional social. Un verdadero enfoque pedagógico de EACD debe proponer la proyección de la formación de una persona y de una sociedad orientada por preguntas como las trazadas por Abramovich (1999):

¿Qué cosas necesitamos saber para enseñar a nuestros alumnos a litigar diligentemente en la defensa de intereses públicos o colectivos? ¿Cómo prepararlos para enfrentar a un Tribunal y al abogado de la otra parte? ¿Cómo ayudarlos a relacionarse con los clientes, a identificar correctamente su voluntad o sus deseos? ¿Qué técnicas debemos suministrarles para que sepan investigar un caso, probar los hechos, argumentar jurídicamente en el marco de un conflicto real? (Abramovich, 1999, p. 61).

A sus interrogantes Abramovich (1999) responde que las Facultades de Derecho "deben recuperar un íntimo contacto con las necesidades de los clientes y con aquello que 
constituye la práctica real de los tribunales y de los abogados" (p. 65), lo que se considera representa asumir el compromiso de trabajar de cara a la realidad social como laboratorio obligado para el Derecho. Así lo explica Medina (2001)

Ser docente es un compromiso social y humano, aún para aquellos abogados y profesores de Derecho con extremado espíritu conservador (...) tienen que salir de las aulas de clase y de los libros, ir a la calle y al foro judicial, leer la realidad (pp. 116 - 117).

Por lo tanto, tomando como referente la enseñanza y aprendizaje de la medicina, la EACD asume al estudiante como eje central del proceso formativo a partir de una relación de horizontalidad con el docente y los demás estudiantes que integran la CJ. Al igual que el estudiante de medicina, el futuro abogado es localizado de forma temprana en escenarios sociales reales, o en su defecto simulados, donde tiene contacto directo con casos prácticos complejos de distinta naturaleza. Esa práctica rompe la parcelación, desintegración y autonomía de las áreas que componen el campo de conocimiento jurídico para afrontar situaciones como objetos que articulan e integran los conocimientos del Derecho y lo trascienden a otras áreas, dando lugar a la enseñanza y el aprendizaje interdisciplinar.

Para el estudiante de Derecho el contacto directo con la realidad social compleja se asemeja al contacto que el estudiante de medicina tiene con los pacientes enfermos. Lo anterior da lugar a un escenario que posibilita equilibrar la teoría y la práctica en la enseñanza y el aprendizaje, generando para el estudiante la necesidad de incorporar los conocimientos teóricos en su ejercicio. Se hace esencial la implementación e indagación de los referentes que facilitan la aprehensión de cada caso y la solución al problema que representa cada uno de ellos, demandando la comprensión preliminar de los hechos, acoger el testimonio y sentir del cliente, y caracterizar la situación sin desechar las variables económicas, políticas y culturales.

La EACD debe llevar al estudiante a un escenario donde se empodere de su proceso formativo a través del debate, el sentido crítico, reflexivo y propositivo en el que las teorías puedan ser contrastadas con los hechos a partir de ejercicios prácticos, aportando sobre el aprendiz la carga emotiva y racional de la fuente empírica presente en el Derecho. Para la realización del citado enfoque pedagógico, este artículo propone la EACD como un enfoque integrado e integrador constituido esencialmente por la $\mathrm{CJ}$ y el método clínico como los dos componentes básicos pero no exclusivos o excluyentes, que sirven de escenario para la incorporación de otros que, por la naturaleza flexible, abierta y dinámica de la EACD, pueden ser incorporados por colectivo clínico de acuerdo a sus proyecciones e impronta diferenciadora para el proceso de formación de los nuevos abogados que se gestan al amparo de su universidad.

\section{3. ¿CÓMO SE ENSEÑA? A PARTIR DE LA CLÍNICA JURÍDICA Y EL MÉTODO CLÍNICO}

Sin la implementación de un método clínico las CJ perderían su identidad y podría degenerar en una expresión tradicional de la formación de los abogados. La CJ se puede definir como un espacio dentro del cual se implementa de forma preponderante el método clínico de EACD. Al constituir el espacio de encuentro para el trabajo colaborativo del colectivo clínico, en ella se dan cita periódicamente docentes y estudiantes para abarcar el estudio del Derecho desde los postulados de la formación clínica. Por ello que la CJ sea 
el lugar para la ejecución del método clínico; que incluye la posibilidad de implementar gran variedad de mecanismos y herramientas prácticas, sin despreciar el conocimiento teórico y despertando en los estudiantes de derecho el compromiso de enfrentar de manera eficaz necesidades imperantes observadas en la sociedad (Vásquez, 2008, p. 11-21).

La CJ es el componente de la EACD en el cual se da:

(...) un ejercicio participativo, de confluencia de opiniones de profesores y estudiantes, en el cual se propone revelar desde el principio el objetivo que se persigue al llevar el caso, el impacto que una decisión judicial en el caso en concreto puede llegar a tener en el sistema legal, los conflictos éticos que debe afrontar el abogado (Castro, 2004, p. 163).

Las CJ "reconocen la necesidad de incluir en sus análisis o estudios de casos aspectos normalmente ignorados o desestimados por los abogados que realizan un ejercicio tradicional del litigio.” (Molina, 2008, p. 194) o como explica Puga (2000) son “(...) procesos pedagógicos innovativos cuyo objetivo es entrenar a los estudiantes de derecho en las destrezas de la práctica profesional y elevarla a niveles de mayor desarrollo y relevancia social." (p. 49).

Por su parte el método clínico, como explican Correa y Vásquez (2008) siguiendo a Frank (1933) surge de la observación de los métodos utilizados en la educación médica, en los cuales, como ya se mencionó, se asegura un paralelismo entre la enseñanza teórica y los mecanismos que proporcionan al estudiante trabajo práctico con situaciones reales. Los profesores Alexander I. Lyublinsky y William Rowe implementaron un trabajo denominado método clínico que, siguiendo las pautas del proceso de formación médica, conlleva para los estudiantes de Derecho adentrarse en las mismas etapas básicas de entrenamiento clínico.

Para nuestra apuesta, el método clínico puede ser definido como el conjunto de pasos articulados secuencialmente entre sí, a través del cual se determina una experiencia de formación adscrita a los derroteros de la EACD, un método que en palabras de Abramovich (1999) le debe asegurar al estudiante: "analizar su actuación y corregir sus errores" (p. 72), toda vez que "si pudiera resumirse en una frase el método clínico, diríamos que procura que el alumno aprenda a pensar el derecho ocupando un lugar o un rol en un conflicto." (Abramovich 1999, p. 79).

Desde la experiencia mayoritaria de las CJ colombianas, el método clínico comienza con la noticia del caso, lo que puede conllevar ejercicios o procedimientos relacionados con su recepción, lo cual es determinado por cada CJ, al igual que los criterios o modalidades que definen la viabilidad y selección de la situación que concentrará el ejercicio. Una vez el caso es seleccionado, los pasos a seguir están definidos por la formulación de un problema que guiará la articulación equilibrada de la teoría y la práctica. A partir de la formulación del problema, posteriormente se hace concertación de los objetivos que guían el proceso clínico, se construye el referencial teórico, jurídico y fáctico del caso, se diseña y proyecta la estrategia de intervención o incidencia, la cual puede ser o no litigiosa. Finalmente tiene lugar la evaluación, preferentemente de tipo colectivo (coevaluación) y personal (autoevaluación). 


\section{EL CARÁCTER DIFERENCIAL DE LA EACD FRENTE A OTRAS MODALIDADES}

Proponer a la EACD como un enfoque pedagógico contribuye a la superación de la tradición y a instalar una impronta diferencial e innovadora en la educación jurídica. No obstante, es preciso reiterar que la enseñanza y el aprendizaje clínico no es exclusiva del campo de conocimiento del derecho, y tampoco excluyente de otros enfoques, modelos y didácticas provenientes de otros campos del saber. En la diversidad de posibilidades la EACD fortalece su identidad como un enfoque diferencial al tiempo que esclarece las relaciones que entabla con esas otras opciones. Dos de ellas llaman la atención de forma particular: la simulación y el aprendizaje basado en problemas (ABP), ambas también con orígenes en la formación médica.

La simulación "consiste en situar a un educando en un contexto que imite algún aspecto de la realidad" (Salas Perea y Ardanza Zuleta, 1995), se concentra en la emulación o representación de situaciones y supuestos que el estudiante podrá encontrar en su ejercicio profesional (Galindo Caldés, 2020, p. 73). De allí que la simulación implica una representación de una realidad que puede ser controlada (Urra Medina et al., 2017), razón por la cual "ofrecer al educando la oportunidad de realizar una práctica análoga a la que realizará en su interacción con la realidad” (Salas Perea y Ardanza Zuleta, 1995).

La simulación comparte varias fortalezas con la EACD. Relega la formación que se sustenta en la teorización, y con ello, en la magistralidad docente, la retórica y la memorización. Al soportar la enseñanza y el aprendizaje en un equilibrio teórico práctico, reposiciona al estudiante como centro de la intención del acto pedagógico, el cual se enfrenta a la asignación de un supuesto fáctico sobre el cual es preciso definir la distribución de roles, reconocer el proceso que rige el supuesto asignado, elaborar escritos de tipo judicial y desplegar la competencia argumentativa y de expresión oral.

De manera que la simulación resignifica el concepto de aula, genera un proceso de formación colectivo y con gran contenido colaborativo en donde el estudiante no es un actor pasivo. Así se reseña, por ejemplo, en la experiencia de la Universidad Rovira i Virgili, donde exaltan que permite trabajar las competencias escrita y oral (Domenech y Casanova, 2014). Y es que la simulación acarrea trabajo en equipo, búsqueda de fuentes de información, capacidad de debate, de redacción, de argumentación y de improvisación, la apropiación de roles, y de la responsabilidad que ellos conllevan (Morales Ortega, 2018). No obstante sus diversos puntos en común con la EACD, entre ambas modalidades hay una diferencia amplia y profunda.

Mientras la EACD es llevada a la tipología de enfoque pedagógico, y con ello, puede fundamentar modelos, la simulación no reúne todos los elementos pedagógicos para llegar al nivel de enfoque, ajustándose más a la calidad de modelo. Lo anterior en la medida que un modelo pedagógico puede ser definido como la "herramienta que se utiliza para describir, decidir e interpretar actitudes en diferentes situaciones, haciendo viable el reproducir hipótesis y elaborar estrategias para su intervención." (Garzón Saladen y Romero González, 2018, p. 312) a lo cual se ajusta más la simulación. Esta no propone principios que permitan recrear el proceso formativo, en la medida que trabaja con realidades controladas, promueve una formación análoga cifrada en la experiencia previa, privilegia la judicialización como ejercicio del derecho, y no se ve obligada a introducir al estudiante como actor autónomo de una realidad compleja particular, lo que la confirma como algo estandarizado y reproducible. 
Mientras la simulación privilegia una enseñanza y aprendizaje del derecho por imitación o representación de la realidad, la EACD privilegia el trabajo con la realidad, no con casos hipotéticos, emulaciones, representaciones o actividades controladas. El estudiante asume el rol de abogado a partir de la confrontación constante con el supuesto fáctico en su devenir histórico, político, social y cultural, sin que medie posibilidad de controlar y redireccionar el fluir de los hechos, de las actuaciones procesales, de la carga afectiva o mediática. De allí que mientras la simulación construye una realidad para formar al abogado, la EACD construye al abogado en la realidad.

La simulación privilegia la formación en la judicialización de los supuestos. Se trata de un modelo que se concentra en las actuaciones y el rigorismo impuesto por la norma para adelantar actuaciones ante las autoridades judiciales, lo que da mayor cabida al trabajo con áreas adscritas al derecho procesal y probatorio, y aminora la interrelación e intercambio con otras áreas del derecho y de otros campos del conocimiento. Por su parte, la EACD incluye la formación para la judicialización de situaciones, pero incluye alternativas como el activismo político, la movilización social, las campañas y estrategias comunicativas, los mecanismos alternativos de solución de controversias, los escenarios de participación democrática, y la investigación para la pedagogía ciudadana.

Mientras la simulación da preponderancia al acto pedagógico cifrado en el Quién es formado y sobre Qué contenidos es formado, la EACD integra a esos componentes el A quién va dirigida esa formación, y el Cómo se procede con el acto pedagógico. Es por ello que a nivel de competencias la EACD no se limita a las de tipo argumentativo, sean escritas y orales, pues también compromete las interpretativas, comprensivas, propositivas y relacionales.

Por su parte, el ABP es una figura altamente compatible con la EACD. En el ABP se presenta el problema de la forma como sucede en la realidad, se identifican las necesidades del aprendizaje, también la información requerida para el caso y se emplea en la búsqueda de una solución (Font Ribas, 2004 citado en Barrio Gallardo, 2015). De allí que el ABP se explique como "un método de aprendizaje basado en el principio de usar problemas como punto de partida para la adquisición e integración de los nuevos conocimientos" (Barrows, 1986), y centra el aprendizaje en el estudiante, el conocimiento se produce en grupos pequeños, los docentes son facilitadores, el problema es el foco de organización y del estímulo para el aprendizaje (Morales Bueno y Landa Fitzgerald, 2004) todos, puntos en común con la EACD.

Sin embargo, frente a la EACD el ABP es un método, y a la par del método de enseñanza clínica del derecho, puede hacer parte del proceso formativo que se ejecuta en una CJ en particular. $\mathrm{Al}$ interior de la EACD el ABP es un componente, más no es la experiencia formativa en su totalidad. Con el valor de su contribución, puede llegar incluso a ser absorbido por el método clínico en supuestos como la triple problematización -social, jurídica e investigativa- sin dejar de lado que el ABP tampoco preferencia la localización del estudiante en un supuesto de la realidad que lo enfrente con el desarrollo de los acontecimientos y la necesidad de la interdisciplinariedad.

\section{DISCUSIÓN Y CONCLUSIONES}

Una última contribución a las formas en cómo las CJ pueden fortalecer el nuevo enfoque de enseñanza y aprendizaje del Derecho se centra en la incorporación de la investigación como herramienta para desplegar el método clínico y otras metodologías activas de aprendizaje. 
De la multiplicidad de elementos, estrategias y derroteros que guían la EACD en las CJ seguro se infiere la presencia de la investigación como una función a la que se acude con frecuencia, en la mayoría de las oportunidades de manera no declarada ni organizada. $\mathrm{Y}$ es que en el ejercicio clínico si bien la labor de investigar es recurrente, la forma en cómo es concebida e incorporada no es necesariamente el resultado de una elaboración previa, declarada y planeada, lo que en oportunidades distorsiona la naturaleza misma de la investigación como actividad y su función en la enseñanza y el aprendizaje a través de la problematización para la generación de conocimiento, o en otros, sustituye el método clínico y afecta la esencia de la EACD.

De lo anterior que sea necesario especificar que el método clínico y el ejercicio de las CJ no es necesariamente un proceso orientado de manera estricta por la investigación como herramienta de validación o generación de conocimiento. Si bien investigar hace parte del ejercicio clínico, como función sustantiva de la educación superior puede cumplir un rol preponderante, planeado y declarado dentro de la EACD que la fortalezca como enfoque pedagógico, sin sustituirla o desnaturalizarla.

Si se recuerda que la investigación representa una actividad humana guiada por una pregunta problematizadora de la que se desprende una serie de pasos articulados entre sí por medio de los cuales se busca la solución de aquella, vemos como entre la investigación y el método clínico hay elementos comunes que no se contraponen, y, por el contrario, se concatenan en un mismo fin. Pero si bien comparten como punto de inicio una pregunta problema, en el método clínico inicialmente ésta debe ser aprehendida como un problema social. Exige la comprensión y dominio de los hechos y de los referentes jurídicos que se contrastan con aquellos; exige su traducción a un problema jurídico, determina y define la orientación de los objetivos de acción del equipo clínico, justifica su actividad, y fija la proyección de la estrategia de actuación. Como piedra angular del método clínico el problema social también puede ser el componente principal de todo proceso de investigación, formativa y científica, al que se articulan objetivos, justificación, hipótesis, referente teórico y diseño metodológico.

El método clínico y la investigación pueden concurrir sobre la realidad social que se concentra en el caso seleccionado por el colectivo clínico. El caso puede ser guiado y ampliando para su comprensión a partir de una pregunta elaborada por la CJ desde una actividad propiamente investigativa, un interrogante adicional y distinto a la pregunta social y a la jurídica. Con base en aquella, puede incorporar técnicas que involucran métodos propios de las ciencias sociales para nutrir desde la aprehensión de la pregunta de investigación cada uno de los momentos del método clínico, y con ello, potenciar el proceso formativo.

La pregunta investigativa subsiste siempre que se justifique a partir de funciones declaradas para el ejercicio clínico, esto es, complementa el proceso de EACD más no lo suplanta, de manera que como manifestación investigativa del problema seleccionado contribuye con la guía del método clínico y además permite la generación de conocimiento. La pregunta investigativa, articulada con las pregunta social y jurídica que tienen lugar en el método clínico, se define por el espíritu de tributar a la solución de la situación social seleccionada por la CJ.

De lo anterior que la formulación del problema y su representación a través de preguntas juegue un papel determinante en la EACD. La comprensión del sustento fáctico en el cual se adscribe el caso debe traducirse en una pregunta que sintetice la problemática 
social, concentrando el primer punto de intervención que guía el ejercicio clínico. Sobre esa pregunta la CJ podrá derivar un segundo problema, de tipo jurídico, a través del cual la realidad delimitada como problemática social se entrelazada con el Derecho y es leída a través de éste aprovechando varias de sus acepciones.

Trascender a la acepción científica del Derecho conlleva adscribirlo en un proceso de investigación a partir del cual se enriquece, amplifica, valida o especializa como campo de conocimiento en el que reposa como construcción científica, pero que bajo la premisa de la superación de las formalidades y las tradiciones acríticas y paquidérmicas que no han facilitado acreditar su función social como conocimiento científico, tiene la labor de permear la realidad de la cual se desprende. Por ello en el método clínico se propone la formulación de un tercer interrogante; en él deben converger el problema social y su traducción a problema jurídico, que englobados como objetos de estudio permiten la implementación del método científico y la generación del conocimiento suficiente para fortalecer las posibles soluciones al problema clínico que guía el proceso de EACD.

La formulación de problemas, uno social, uno jurídico, y otro investigativo, todos sustentados sobre la misma realidad, permiten diseñar una modalidad de enseñanza y aprendizaje en la cual, a la experiencia formativa soportada en el equilibrio teórico práctico del estudiante, se incorpora la pregunta como herramienta privilegiada del proceso de formación.

El enfoque pedagógico de EACD debe ser una contribución al posacuerdo colombiano, y con ello, a la recreación de la cultura jurídica que busca transitar a nuevos escenarios. Mientras el problema jurídico debe conseguir el potencial de englobar la realidad social compleja para asegurar la proyección de una estrategia que impulsa acciones encaminadas a la solución del problema empleando mecanismos e instrumentos jurídicos, políticos y/o sociales, el problema de investigación incorpora esta función sustantiva como un proceso de contribución al tratamiento y solución del problema social y jurídico, toda vez que: "El problema jurídico constituye un verdadero problema de investigación necesariamente teórico, nace y se debe al problema social, el cual le da contenido y estructura" (Correa y Vásquez, 2009, p. 139). Como pocas veces, realidad, Derecho y ciencia se encuentran de una manera tan íntima; el desafío debe ser su consolidación a través de su implementación con el enfoque pedagógico propuesto.

\section{REFERENCIAS BIBLIOGRÁFICAS}

Abramovich, V. E. (1999). La enseñanza del derecho en las clínicas legales de interés público. Materiales para una agenda temática. En: Defensa jurídica del interés público. Enseñanza, estrategias, experiencias. Santiago de Chile: Universidad Diego Portales.

BarrowS H. (1996) Problem-Based learning in medicine and beyond: A brief overview. In Wilkerson L., Gijselaers W. H. (eds). Bringing Problem-Based Learning to Higher 157 Education: Theory and Practice. San Francisco: Jossey-Bass Publishers, 3-12.

Barrio Gallardo, A. (2015) El ABP en derecho Privado: un proyecto de innovación docente. Academia, Revista sobre Enseñanza del Derecho, 13(25), 131-151.

Barrows, H. (1986) A taxonomy of problem based learning methods. Medical Education, 20(6), 481-486.

Behar, P. A., Passerino, L. y Bernardi, M. (2007). Modelos pedagógicos para a educação a distancia: pressupostos teóricos para a construçao de objetos de aprendizagem. Revista Renote, 5(2). https://doi.org/10.22456/1679-1916.14242

Bobbio, N. (2015). El problema del positivismo jurídico. México: Fontamara. 
Bohmer, M. F. (2003). Algunas sugerencias para escapar del silencio del aula. Revista sobre enseñanza del Derecho 1(1), 13-34.

Castillo Cedeño, I., Flores Davis, L. E., Jiménez Corrales, R. E. y Perearnau Torras, M. (2008). Una reflexión necesaria: Posibilidad de la construcción de un modelo pedagógico en la educación superior. Revista Electrónica Educare, XII(1), 123-34.

Castro Buitrago, E. (2004). Perspectivas de la enseñanza clínica del Derecho en Colombia. Revista Opinión Jurídica 3(5), 161-68.

(2015). Un modelo de estrategia didáctica para la enseñanza clínica del derecho con énfasis en investigación. Revista Indisciplinas, 1(1), 69-98.

Cañaveral, W. y Vásquez Santamaría, J. E. (2010) Modelos pedagógicos: Referentes para constituir una propuesta pedagógica para la formación de los profesionales del Derecho en Colombia. Revista Facultad de Derecho Fundación Universitaria Luis Amigó, (3), 76-89.

Cepeda Jiménez, J. A. (2016). El posacuerdo en Colombia y los nuevos retos de la seguridad. Cuadernos de estrategia, (18), 125-294. Recuperado de: https://dialnet.unirioja.es/servlet/ articulo?codigo $=5673544$

Coll, C. (1991). Aprendizaje escolar y construcción del conocimiento. Buenos Aires: Paidós.

Correa Montoya, L. y Vásquez Santamaría, J. E. (2008). La enseñanza clínica del Derecho: Transformando la forma de enseñar y ejercer el Derecho. Revista Studiositas 3(1), 34-40.

(2009) Investigación jurídica por medio de la enseñanza clínica del Derecho. En: La Enseñanza Clínica del Derecho. Compilado por Lucas Correa Montoya (pp. 129-58). Medellín: Fundación Universitaria Luis Amigó.

De Zubiría Samper, M. (1999). Estructura de la pedagogía conceptual. En: Pedagogía conceptual. Desarrollos psicológicos, pedagógicos y psicológicos. Bogotá: Fondo de Publicaciones Bernardo Herrera Merino, FIPCAM. (Tratado de Pedagogía Conceptual, 7).

De Zubiría Samper, J. (2006). Los modelos pedagógicos: Hacia una pedagogía dialogante. Bogotá: Cooperativa Editorial Magisterio.

Domenech, F. A. y Casanova Martí, R. (2014) La práctica de juicios en el grado de Derecho. Redica (Derecho) Serie Derecho Procesal, 5(1), 307-317.

Duarte, E. (2012). La enseñanza en el ambiente clínico: principios y métodos. Revista de Docencia Universitaria, (10), 149-75.

Fergusson Talero, A. M. (2009). La enseñanza del derecho en Colombia. En: La Enseñanza Clínica del Derecho. pp. 21-66. Medellín: Fundación Universitaria Luis Amigó.

Flórez Ochoa, R. (1997) Hacia una pedagogía del conocimiento. Bogotá: Mc Grawn Hill.

Frank, J. (1933). Why not a Clínical Lawyer-School? En: Hunder, A. et al. (1997) Clinical Anthology. Readings for Live-Client Clinics. Cincinnati: Anderso’s Law School Publications.

Galindo Caldés, R. (2020) La simulación en derecho de la función pública: representación sindical y negociación colectiva. Revista de la Facultad de Derecho de México, LXX(278), 71-90.

Garzón-Saladen, A. \& Romero-González, Z. (2018). Los modelos pedagógicos y su relación con las concepciones del derecho: puntos de encuentro con la educación en derecho. Rev. Investig. desarro. innov, 8(2), 311-320. doi: 10.19053/20278306.v8.n2.2018.7968

Gómez Agudelo, G. A. (2008) La pedagogía tradicional en las facultades de Derecho en Colombia. Revista Via Iuris, (5), 105-109.

Goyez Moreno, I. (2010). Acreditación y calidad en la educación superior colombiana. En: Los abogados en Colombia (pp. 90-102). Bogotá, Colombia: Universidad Nacional de Colombia.

Hernández Rojas, G. (2005) El aprendizaje basado en problemas. En: Enfoques pedagógicos y didácticas contemporáneas. Bogotá: Fundación Internacional de la Pedagogía Conceptual.

Khun, T. (2004) La estructura de las revoluciones científicas. México: Fondo de Cultura Económica ( $\left.5^{\mathrm{a}} \mathrm{Ed}.\right)$.

Londoño Toro, B. (2015). Educación legal clínica y litigio estratégico en Iberoamérica. Bogotá: Universidad del Rosario. 
Medina Torres, C. B. (2001) Proceso Modelo de Stuttgart y Enseñanza del Derecho Procesal. Bogotá: Ediciones Doctrina y Ley Ltda.

Molina Saldarriaga, C. A. (2008). "Fundamentos teóricos y metodológicos del Método Clínico de enseñanza del derecho". Revista Facultad de Derecho y Ciencias Políticas 38(108), 187-213.

Morales Bueno, P. y Landa Fitzgerald, V. (2004) Aprendizaje basado en problemas. Theoria, 13, pp. 145-157.

Morales Ortega, J. M. (2018) Dramatización del conocimiento: simulaciones en contexto de los estudios jurídicos laborales de grado y de posgrado. Revista Jurídica de Investigación e Innovación Educativa, (17), 81-98.

Nino, C. S. (2003). Introducción al análisis del Derecho. Buenos Aires: Editorial Astrea.

Not, L. (1983). Las pedagogías del conocimiento. México: Fondo de la Cultura Económica.

Pabón Mantilla, A. P., Aguirre Román, J. O. y Cáceres Rojas, P. B. (2016). La clínica jurídica como estrategia para fortalecer las competencias ciudadanas: Una apuesta por la convivencia pacífica. Revista Ratio Juris, 11(23), 27-46.

Pérez Mejía, M. A. (2016). Post-acuerdo y objetivos de desarrollo sostenible: educación para la paz, una oportunidad para Colombia propuesta de curso de verano en la universidad Eafit, basada en el barco de la paz. Medellín: Universidad Eafit. Recuperado de: https://repository.eafit.edu. co/bitstream/handle/10784/8724/MariaAntonia_PerezMejia_2016.pdf?sequence=2

Prieto Sanchís, L. (2005). Apuntes de teoría del Derecho. Madrid: Editorial Trotta.

Puga, M. (2000) Los desafíos de las clínicas jurídicas en Argentina. En: F. González (ed.) Cuadernos de Análisis Jurídico. Santiago: Universidad Diego Portales.

Salas Perea, R. y Ardanza Zuleta, P. (1995) La simulación como método de enseñanza y aprendizaje. Educación Médica Superior, 9(1).

Sarmiento, J. P. (2014). La educación jurídica colombiana y la globalización: Entre los estudios de "cajanegra", el formalismo jurídico y la nueva hegemonía. International Law: Revista Colombiana de Derecho Internacional, (24), 59-82.

Torres Villareal, M. L. (2013) La enseñanza clínica del derecho: una forma de educación para el cambio social. La experiencia del grupo de acciones públicas de la Universidad del Rosario. Revista de la Facultad de Derecho y Ciencias Políticas 49(119), 705-734.

Urra Medina, E., Sandoval Barrientos, S. y Irribarren Navarro, F. (2017) El desafío y futuro de la simulación como estrategia de enseñanza en enfermería. Investigación en Educación Médica, 6(22), 119-125.

Vásquez Santamaría, J. E. (2008) Fundamentos para la creación de una clínica jurídica en la Universidad Católica Luis Amigó como apoyo en la enseñanza práctica del derecho. Memorias CIIEC, 2(3), 11-21. . (2012). Modelos, actores y corrientes del Derecho en Colombia. Medellín: Universidad . (2013). Indisciplinar el Derecho. Investigación jurídica y sociojurídica en Colombia.

Medellín: Universidad Autónoma Latinoamericana. 
